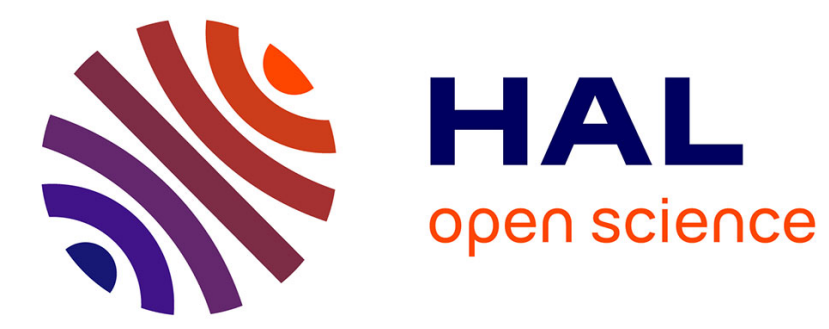

\title{
Rayleigh Time-varying Channel Complex Gains Estimation and ICI Cancellation in OFDM Systems
}

\author{
Hussein Hijazi, Laurent Ros
}

\section{To cite this version:}

Hussein Hijazi, Laurent Ros. Rayleigh Time-varying Channel Complex Gains Estimation and ICI Cancellation in OFDM Systems. European Transactions on Telecommunications, 2009, 20 (8), pp.782796. 10.1002/ett.1366 . hal-00373794

\section{HAL Id: hal-00373794 https://hal.science/hal-00373794}

Submitted on 7 Apr 2009

HAL is a multi-disciplinary open access archive for the deposit and dissemination of scientific research documents, whether they are published or not. The documents may come from teaching and research institutions in France or abroad, or from public or private research centers.
L'archive ouverte pluridisciplinaire HAL, est destinée au dépôt et à la diffusion de documents scientifiques de niveau recherche, publiés ou non, émanant des établissements d'enseignement et de recherche français ou étrangers, des laboratoires publics ou privés. 


\title{
Rayleigh Time-varying Channel Complex Gains Estimation and ICI Cancellation in OFDM Systems
}

\author{
Hussein Hijazi and Laurent Ros \\ E-mail: hussein.hijazi@gipsa-lab.inpg.fr, laurent.ros@gipsa-lab.inpg.fr \\ Tel: +33 (0)4 76827178 and Fax: +33(0)4 76826384 \\ GIPSA-lab, Departement Image Signal \\ BP 46 - 38402 Saint Martin d'Hères - FRANCE
}

\begin{abstract}
SUMMARY
In this paper, we consider an orthogonal-frequency-division-multiplexing (OFDM) mobile communication system operating in downlink mode in a time-varying multipath Rayleigh channel scenario. We present a Mean Square Error theoretical analysis for a multipath channel complex gains estimation algorithm with inter-sub-carrier-interference (ICI) reduction using a comb-type pilot. Assuming the presence of delayrelated information, the time average of the multipath complex gains, over the effective duration of each OFDM symbol, are estimated using LS criterion. After that, the time-variation of the multipath complex gains within one OFDM symbol are obtained by interpolating the time-averaged symbol values using lowpass interpolation. Hence, the channel matrix, which contains the channel frequency response and the coefficients of ICI, can be computed and the ICI can be reduced by using successive interference suppression (SIS) in data symbol detection. The algorithm's performance is further enhanced by an iterative procedure, performing channel estimation and ICI suppression at each iteration. Theoretical analysis and simulation results show a significant performance improvement for high normalized Doppler spread (especially after the first iteration) in comparison to conventional methods.
\end{abstract}

\section{INTRODUCTION}

ORTHOGONAL frequency division multiplexing (OFDM) is widely known as the promising communication technique in the current broadband wireless mobile communication system due to the high spectral efficiency and robustness to the multipath interference. Currently, OFDM has been adapted to the digital audio and video broadcasting (DAB/DVB) system, highspeed wireless local area networks (WLAN) such as IEEE802.11x, fixed wireless access WiMax IEEE802.11e, 3GPP/LTE, HIPERLAN II and multimedia mobile access communications (MMAC), ADSL, digital multimedia broadcasting (DMB) system and multi-band OFDM type

Part of this work was presented in 50-th IEEE GLOBECOM, Washington, USA, November 2007 [1] and in European Wireless Conference (EW), Paris, FRANCE, April 2007 [2] ultra-wideband (MB-OFDM UWB) system, etc. However, OFDM system is very vulnerable when the channel changes within one OFDM symbol. In such case, the orthogonality between subcarriers are easily broken down resulting the inter-sub-carrier-interference (ICI) so that system performance may be considerably degraded.

A dynamic estimation of channel is necessary since the radio channel is frequency selective and time-varying for wideband mobile communication systems [5] [17] [19]. In practice, the channel may have significant changes even within one OFDM symbol. In this case, it is thus preferable to estimate channel by inserting pilot tones into each OFDM symbol which called comb-type pilot [6]. Assuming insertion of pilot tones into each OFDM symbols, the conventional channel estimation methods consist generally of estimating the channel at pilot frequencies and next interpolating the channel 
frequency response. The estimation of the channel at the pilot frequencies can be based on Least Square (LS) or Linear Minimum Mean-Square-Error (LMMSE). LMMSE has been shown to have better performance than LS [6]. In [7], the complexity of LMMSE is reduced by deriving an optimal low-rank estimator with singularvalue-decomposition. The interpolation techniques used in channel estimation are linear interpolation, second order interpolation, low-pass interpolation, spline cubic interpolation, time domain interpolation and Wiener filtering as 2-D interpolation [19]. In [8], low-pass interpolation has been shown to perform better than all the interpolation techniques. In [9] the channel estimator is based on a parametric channel model, which consists of estimating directly the time delays and complex attenuations of the multipath channel. This estimator yields the best performance among all comb-type pilot channel estimators, with the assumption that the channel is invariant within one OFDM symbol. For fast timevarying channel, many existing works resort to estimate the equivalent discrete-time channel taps which are modeled in a linear fashion [17] or more generally by a basis expansion model (BEM) [15] [16]. The BEM methods [15] used to model the equivalent discrete-time channel taps are Karhunen-Loeve BEM (KL-BEM), prolate spheroidal BEM (PS-BEM), complex-exponential BEM (CE-BEM) and polynomial BEM (P-BEM).

In the present paper, we present an iterative algorithm for channel estimation with inter-sub-carrier-interference (ICI) reduction in OFDM downlink mobile communication systems using comb-type pilots. Our interesting is to estimate directly the physical channel instead of the equivalent discrete-time channel. That means estimating the physical propagation parameters such as multipath delays and multipath complex gains. By exploiting the nature of Radio-Frequency channels, the delays are assumed to be invariant (over several OFDM symbols) and perfectly estimated, and only the complex gains of the multipath channel have to be estimated as we have already done in CDMA context [3] [4]. Notice that an initial very performant multipath time delays estimation can be obtained by using the ESPRIT (estimation of signal parameters by rotational invariance techniques) method [9] [11]. For a Jakes' spectrum Rayleigh gain, we have showed that the central value and the time averaged value over one OFDM symbol are extremely closed even for high realistic Doppler spread. So, for a block of OFDM symbols, we propose to estimate the time average of the complex gains, over the effective duration of each OFDM symbol of different paths, using LS criterion. After that, the time variation of the different paths complex gains within one OFDM symbol are obtained by interpolating the time averaged symbol values using low-pass interpolation. Hence, the channel matrix, which contains the channel frequency response and the coefficients of ICI, can be computed and the ICI can be reduced by using successive interference suppression (SIS) in data symbol detection. The present proposed algorithm, with less number of pilots and without suppression of interference, gives a good performance over the conventional methods and performs better with starting interference suppression. This proposed algorithm can in fact be considered as a simple extension of an algorithm for time-invariant channels but which brings, as we will show, a significant gain in case of time-varying channels with realistic Doppler spreads. Moreover, we give a theoretical and simulated Mean Square Error (MSE) multipath channel complex gains estimation analysis in terms of the normalized (by the OFDM symbol-time) Doppler spread. This further demonstrates the effectiveness of the proposed algorithm.

This paper is organized as follows. Section II introduces the OFDM baseband model and section III covers the multipath complex gains estimation and the iterative algorithm. Next, Section IV presents some simulation results that demonstrate our technique. Finally, we conclude the paper in Section V.

Notation: Superscripts $(\cdot)^{T}$ and $(\cdot)^{H}$ stand for transpose and Hermitian operators, respectively. $|\cdot|, \operatorname{Tr}(\cdot)$ and $E[\cdot]$ are the determinant, trace and expectation operations, respectively. $\|\cdot\|$ and $(\cdot)^{*}$ are the magnitude and conjugate of a complex number, respectively. - and $\doteq$ denote a vector and a matrix, respectively. $\mathrm{A}[\mathrm{m}]$ denotes the $m$ th entry of the vector $\underline{A}$ and $\mathrm{A}[\mathrm{m}, \mathrm{n}]$ denotes the $[m, n]$ th entry of the matrix $\underline{\underline{A}} . \underline{I}_{N}$ is a $N \times N$ identity matrix and $\operatorname{diag}\{\underline{A}\}$ is a diagonal matrix. $J_{0}(\cdot)$ and $J_{1}(\cdot)$ denote the zeroth-order and the first-order Bessel functions of the first kind, respectively. $\otimes$ denotes the convolution. $\delta_{k, m}$ denotes the Kronecker symbol and $(k)_{N}$ stands for the residue of $\mathrm{k}$ modulo $\mathrm{N}$. Letters between brackets $[d]$ or parentheses $(n)$ denote that $d$ and $n$ are indexes or variables.

\section{SYSTEM MODEL}

Suppose that the symbol duration after serial-to-parallel ( $\mathrm{S} / \mathrm{P})$ conversion is $T_{u}$. The entire signal bandwidth is covered by $\mathrm{N}$ subcarriers, and the space between two neighboring subcarriers is $1 / T_{u}$. Denoting the sampling time by $T_{s}=T_{u} / N$, and assuming that the length of the cyclic prefix is $T_{g}=N_{g} T_{s}$ with $N_{g}$ being an integer. The 
duration of an OFDM symbol is $T=\left(N+N_{g}\right) T_{s}$. In an OFDM system, the transmitter usually applies an $N$-point IFFT to data block normalized QAM-symbols $\left\{X_{(n)}[k]\right\}$ (i.e., $E\left[X_{(n)}[k] X_{(n)}[k]^{*}\right]=1$ ), where $n$ and $k$ represent respectively the OFDM symbol index and the subcarrier index, and adds the cyclic prefix (CP), witch is a copy of the last samples of the IFFT output, to avoid inter-symbolinterference (ISI) caused by multipath fading channels. In order to limit the periodic spectrum of the discrete time signal at the output of the IFFT, we use an appropriate analog transmission filter $G_{t}(f)$. As a result, the output baseband signal of the transmitter can be represented as [9] [1]:

$$
x(t)=\sum_{n=-\infty}^{\infty} \sum_{d=-N_{g}}^{N-1} x_{(n)}[d] g_{t}\left(t-d T_{s}-n T\right)
$$

where $g_{t}(t)$ is the impulse response of the transmission analog filter and $x_{(n)}[d]$, with $d \in\left[-N_{g}, N-1\right]$, are the $\left(N+N_{g}\right)$ samples of the IFFT output and the cyclic prefix of the $n$th OFDM symbol given by:

$$
x_{(n)}[d]=\frac{1}{N} \sum_{m=-\frac{N}{2}}^{\frac{N}{2}-1} X_{(n)}[m] e^{j 2 \pi \frac{m d}{N}}
$$

It is assumed that the signal is transmitted over a multipath Rayleigh fading channel characterized by:

$$
h(t, \tau)=\sum_{l=1}^{L} \alpha_{l}(t) \delta\left(\tau-\tau_{l} T_{s}\right)
$$

where $L$ is the total number of propagation paths, $\alpha_{l}$ is the $l$ th complex gains of variance $\sigma_{\alpha_{l}}^{2}$ and $\tau_{l}$ is the $l$ th delay normalized by the sampling time $\left(\tau_{l}\right.$ is not necessarily an integer). $\left\{\alpha_{l}(t)\right\}$ are wide-sense stationary (WSS) narrow-band complex Gaussian processes with the so-called Jakes' power spectrum of maximum Doppler frequency $f_{d}$ [10] and uncorrellated with respect to each other. The average energy of the channel is normalized to one (i.e., $\sum_{l=1}^{L} \sigma_{\alpha_{l}}^{2}=1$ ). At the receiver side, after passing to discrete time through low pass filtering and A/D conversion, the $\mathrm{CP}$ is removed assuming that its length is no less than the maximum delay. Afterwards, a $N$-point FFT is applied to transform the sequence into frequency domain. The $k$ th subcarrier output of FFT during the $n$th OFDM symbol is given by (see Appendix A):

$$
Y_{(n)}[k]=\sum_{m=-\frac{N}{2}}^{\frac{N}{2}-1} X_{(n)}[m] G_{t}[m] G_{r}[m] H_{(n)}[k, m]+W_{(n)}[k]
$$

where $W_{(n)}[k]$ is white complex Gaussian noise with variance $\sigma^{2}, G_{t}[m]$ and $G_{r}[m]$ are the transmitter and receiver filter frequency response values at the $m$ th transmitted subcarrier frequency, and $H_{(n)}[k, m]$ are the coefficients of the channel matrix from the $m$ th transmitted subcarrier frequency to the $k$ th received subcarrier frequency, and given by (see Appendix A):

$H_{(n)}[k, m]=\frac{1}{N} \sum_{l=1}^{L}\left[e^{-j 2 \pi \frac{m}{N} \tau_{l}} \sum_{q=0}^{N-1} \alpha_{l}^{(n)}\left(q T_{s}\right) e^{j 2 \pi \frac{m-k}{N} q}\right]$

where $k, m \in\left[-\frac{N}{2}, \frac{N}{2}-1\right]$ and $\left\{\alpha_{l}^{(n)}\left(q T_{s}\right)\right\}$ is the $T_{s}$ spaced sampling of the $l$ th complex gain during the $n$th OFDM symbol.

If we assume $N$ transmission subcarriers within the flat region of the frequency response of each of the transmitter and receiver filters, then, by using the matrix notation and omitting the index time $n$, (4) can be rewritten as [1] [2]:

$$
\underline{Y}=\underline{\underline{H}} \underline{X}+\underline{W}
$$

where $G_{t}[m]$ and $G_{r}[m]$ are assumed to be equal to one at the flat region, where $\underline{X}, \underline{Y}, \underline{W}$ are $N \times 1$ vectors given by:

$$
\begin{aligned}
\underline{X} & =\left[X\left[-\frac{N}{2}\right], X\left[-\frac{N}{2}+1\right], \ldots, X\left[\frac{N}{2}-1\right]\right]^{T} \\
\underline{Y} & =\left[Y\left[-\frac{N}{2}\right], Y\left[-\frac{N}{2}+1\right], \ldots, Y\left[\frac{N}{2}-1\right]\right]^{T} \\
\underline{W} & =\left[W\left[-\frac{N}{2}\right], W\left[-\frac{N}{2}+1\right], \ldots, W\left[\frac{N}{2}-1\right]\right]^{T}
\end{aligned}
$$

and $\underline{H}$ is a $N \times N$ channel matrix, which contains the time average of the channel frequency response $H[k, k]$ on its diagonal and the coefficients of the inter-carrier interference (ICI) $H[k, m]$ for $k \neq m$. Notice that $\underline{H}$ would be obviously a diagonal matrix if the complex gains were time-invariant within one symbol.

\section{MULTIPATH COMPLEX GAINS ESTIMATION AND THE ITERATIVE ALGORITHM}

In this section, we propose a method based on comb-type pilots and multipath time delays information to estimate the sampled complex gains $\left\{\alpha_{l}\left[q T_{s}\right]\right\}$ with sampling period $T_{s}$. 


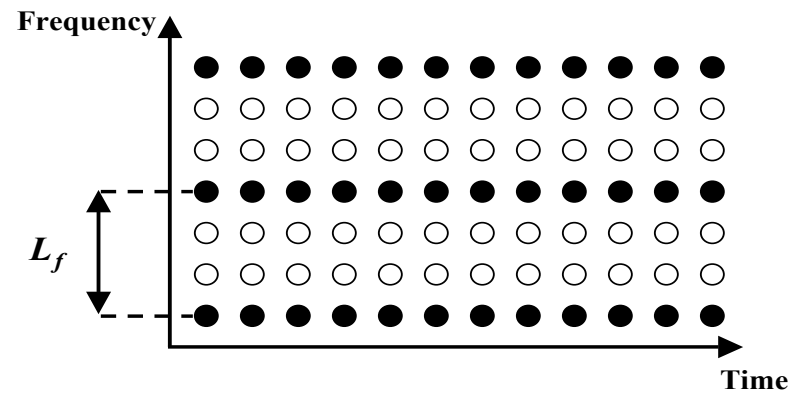

Figure 1. Comb-Type Pilot Arrangement with $L_{f}=3$

\subsection{Pilot Pattern and Received Pilot Subcarriers}

The $N_{p}$ pilot subcarriers are fixed during transmission and evenly inserted into the $N$ subcarriers as shown in Fig. 1 where $L_{f}$ denotes the interval in terms of the number of subcarriers between two adjacent pilots in the frequency domain. $L_{f}$ can be selected without the need of respecting the sampling theorem (in frequency domain) as opposed to the methods shown in [9] [8]. However, as we will see with equation (13), $N_{p}$ must fulfill the following requirement: $N_{p} \geq L$.

Let $\mathcal{P}$ denote the set that contains the index positions of the $N_{p}$ pilot subcarriers defined by:

$$
\mathcal{P}=\left\{p_{s} \mid p_{s}=s L_{f}-\frac{N}{2}, s=0, \ldots, N_{p}-1\right\}
$$

The received pilot subcarriers can be written as the sum of three components [2]:

$$
\underline{Y_{p}}=\underline{\underline{X_{p}}} \underline{H_{p}}+\underline{\underline{H_{p_{I}}}} \underline{X}+\underline{W_{p}}
$$

where the $N_{p} \times N_{p}$ diagonal matrix $\underline{X_{p}}$, and the $N_{p} \times 1$ vectors $Y_{p}$ and $W_{p}$ are given by:

$$
\begin{aligned}
\underline{\underline{X_{p}}} & =\operatorname{diag}\left\{X\left[p_{0}\right], X\left[p_{1}\right], \ldots, X\left[p_{N_{p}-1}\right]\right\} \\
\underline{\underline{Y_{p}}} & =\left[Y\left[p_{0}\right], Y\left[p_{1}\right], \ldots, Y\left[p_{N_{p}-1}\right]\right]^{T} \\
\underline{W_{p}} & =\left[W\left[p_{0}\right], W\left[p_{1}\right], \ldots, W\left[p_{N_{p}-1}\right]\right]^{T}
\end{aligned}
$$

$H_{p}$ is a $N_{p} \times 1$ vector and $H_{p_{I}}$ is a $N_{p} \times N$ matrix with elements given by:

$$
\begin{aligned}
H_{p}\left[p_{s}\right] & =H\left[p_{s}, p_{s}\right]=\sum_{l=1}^{L} \overline{\alpha_{l}} e^{-j 2 \pi \frac{p_{s}}{N} \tau_{l}} \\
H_{p_{I}}\left[p_{s}, m\right] & = \begin{cases}H\left[p_{s}, m\right] & \text { if } m \in\left[-\frac{N}{2}, \frac{N}{2}-1\right]-\mathcal{P} \\
0 & \text { if } m \in \mathcal{P}\end{cases}
\end{aligned}
$$

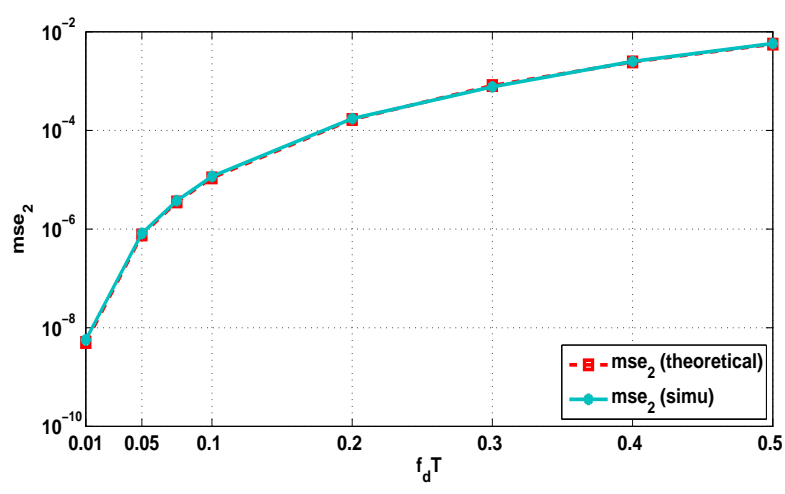

Figure 2. MSE between $\underline{\alpha}_{c}$ and $\underline{\bar{\alpha}}$ for $N=128$

$$
\text { with } \overline{\alpha_{l}}=\frac{1}{N} \sum_{q=0}^{N-1} \alpha_{l}\left(q T_{s}\right)
$$

$\overline{\alpha_{l}}$ is the time average over the effective duration of the OFDM symbol of the $l$ th complex gain. The first component is the desired term without ICI and the second component is the ICI term. $H_{p}$ can be writen as the Fourier transform for the different complex gains time average $\left\{\overline{\alpha_{l}}\right\}$ :

$$
\underline{H_{p}}=\underline{\underline{F_{p}}} \underline{\underline{\alpha}}
$$

where $F_{p}$ and $\underline{\bar{\alpha}}$ are the $N_{p} \times L$ Fourier transform matrix and the $\overline{\bar{N}}_{p} \times 1$ vector, respectively, given by:

$$
\begin{gathered}
\underline{\underline{F_{p}}}=\left[\begin{array}{ccc}
e^{-j 2 \pi \frac{p_{0}}{N} \tau_{1}} & \ldots & e^{-j 2 \pi \frac{p_{0}}{N} \tau_{L}} \\
\vdots & \ddots & \vdots \\
e^{-j 2 \pi \frac{p_{N_{p}-1}}{N} \tau_{1}} & \cdots & e^{-j 2 \pi \frac{p_{N_{p}-1}}{N} \tau_{L}}
\end{array}\right] \\
\underline{\bar{\alpha}}=\left[\overline{\alpha_{1}}, \ldots, \overline{\alpha_{L}}\right]^{T}
\end{gathered}
$$

\subsection{Estimation of Multipath Complex Gains}

For a block $\left\{\alpha_{l}\left(q T_{s}\right), q=0, \ldots, N-1\right\}$ of $\mathrm{N} T_{s}$-spaced sampling of a gaussian complex gains with a frequency of the Jakes' power spectrum $f_{d}$, we have shown in Appendix B that:

a) The $T_{s}$-spaced sampling of the complex gain taken in the middle of the effective duration of the OFDM symbol $\alpha_{l}\left(\frac{N}{2} T_{s}\right)$ is closest to the complex gain time average over the effective duration $\overline{\alpha_{l}}$ defined in (9)

b) For the whole $\mathrm{L}$ gains, the mean square error (MSE) between exact averaged values $\underline{\bar{\alpha}}=\left[\overline{\alpha_{1}}, \ldots, \overline{\alpha_{L}}\right]^{T}$ and exact central values $\underline{\alpha}_{c}=\left[\alpha_{1}\left(\frac{N}{2} T_{s}\right), \ldots, \alpha_{L}\left(\frac{N}{2} T_{s}\right)\right]^{T}$ is 


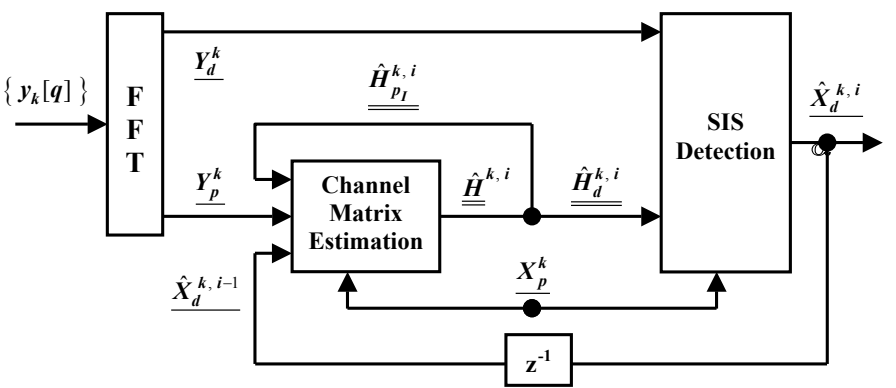

(a)

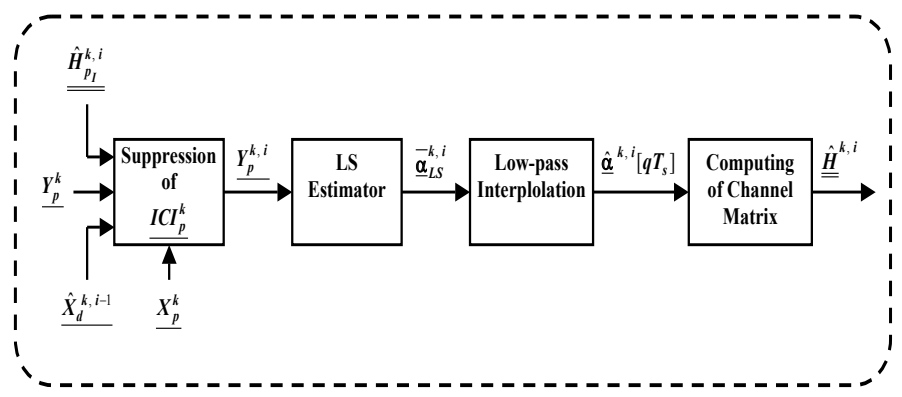

(b)

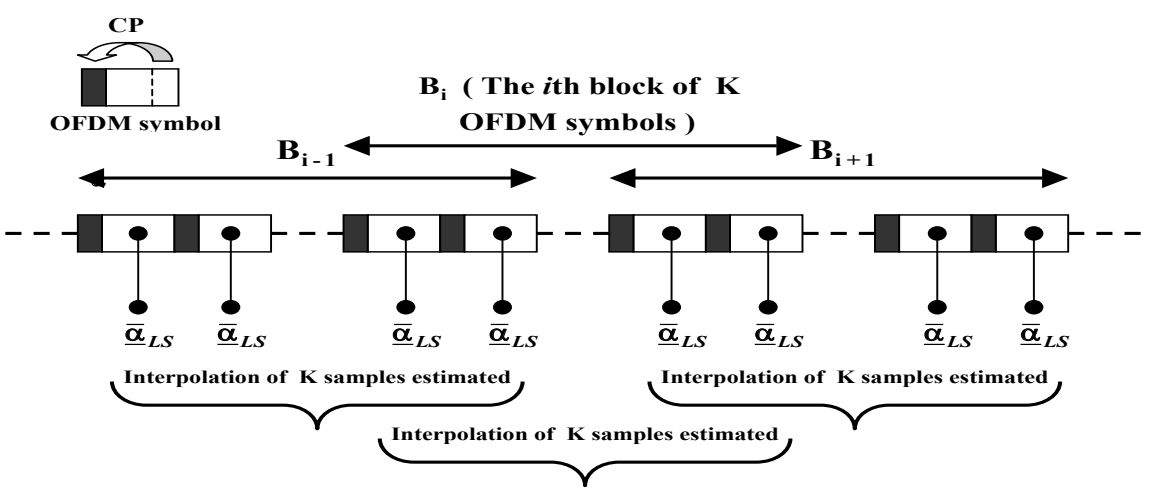

(c)

Figure 3. The block diagrams of the iterative algorithm: (a) the overall channel estimator and ICI suppression block diagram; (b) the channel matrix estimation block diagram; and (c) the diagram of complex gains estimator

given by:

$$
\begin{aligned}
\text { mse }_{2}= & E\left[\left(\overline{\underline{\alpha}}-\underline{\alpha}_{c}\right)^{H}\left(\underline{\bar{\alpha}}-\underline{\alpha}_{c}\right)\right] \\
= & \sum_{l=1}^{L} \sigma_{\alpha_{l}}^{2}\left(\frac{1}{N^{2}} \sum_{q_{1}=0}^{N-1} \sum_{q_{2}=0}^{N-1} J_{0}\left(2 \pi f_{d} T_{s}\left(q_{1}-q_{2}\right)\right)\right. \\
& \left.-\frac{2}{N} \sum_{q=0}^{N-1} J_{0}\left(2 \pi f_{d} T_{s}\left(q-\frac{N}{2}\right)\right)+1\right)
\end{aligned}
$$

Notice that, for a normalized channel, $m s e_{2}$ depends only on $f_{d} T$.

Fig. 2 shows the evolution of $m s e_{2}$ with $f_{d} T$, obtained theoretically from (12) and by Monte-Carlo simulation. We can conclude that, for realistic normalized Doppler spread $\left(f_{d} T<0.5\right)$, the distance between $\underline{\alpha}_{c}$ and $\underline{\bar{\alpha}}$ is very negligible. Hence, we can assume that an estimation of $\underline{\bar{\alpha}}$ is an estimation of $\underline{\alpha}_{c}$. So, by estimating $\underline{\bar{\alpha}}$ for some OFDM symbols and interpolating them by a factor $\left(N+N_{g}\right)$ using low-pass interpolation [8], we obtain an estimation of the sampled complex gains $\left\{\alpha_{l}\left(q T_{s}\right)\right\}$ at time $T_{s}$ during these OFDM symbols, for each path.

The time average of the complex gains, over the effective duration of each OFDM symbol for the different paths, are estimated using the LS criterion. By neglecting the ICI contribution, the LS-estimator of $\underline{\bar{\alpha}}$, which minimizes $\left(\underline{Y_{p}}-\underline{X_{p}} \underline{F_{p}} \underline{\bar{\alpha}}\right)^{H}\left(\underline{Y_{p}}-\underline{X_{p}} \underline{F_{p}} \underline{\bar{\alpha}}\right)$, is represented by:

$$
\begin{aligned}
\underline{\bar{\alpha}}_{L S} & =\underline{\underline{M}} \underline{Y_{p}} \\
\text { with } \underline{\underline{M}} & =\left(\underline{\underline{F_{p}}} \underline{\underline{X_{p}}} \underline{\underline{X_{p}}} \underline{\underline{F_{p}}}\right)^{-1} \underline{\underline{F_{p}}} \underline{\underline{X_{p}}}{ }^{H}
\end{aligned}
$$

It should be noted that, the matrix $F_{p}{ }^{H} X_{p}{ }^{H} X_{p} F_{p}$ in the expression of $\underline{\underline{M}}$ (13) is not invertible if $\overline{\overline{N_{p}}} \overline{\overline{<L}}, \overline{\overline{\text { since }}} F_{p}$ is a matrix of size $N_{p} \times L$.

\subsection{Iterative Algorithm}

The iterative algorithm of channel estimation and ICI suppression is shown in Fig. 3. The whole algorithm is divided into two modes: channel matrix estimation mode and detection mode, as shown in Fig. 3(a). The first mode includes estimating the sampled complex gains $\left\{\alpha_{l}\left(q T_{s}\right)\right\}$ at time $T_{s}$ via LS-estimator and low-pass interpolation and computing the channel matrix as shown in Fig. 3(b). The second mode includes the detection of data symbols by using successive data interference suppression (SIS) 
scheme with one tap frequency equalizer (see Appendix E). A feedback technique is used between these two modes, performing iteratively ICI suppression and channel matrix estimation. In this iterative algorithm, the OFDM symbols are grouped in blocks of $K$ OFDM symbols each one. Each two consecutive blocks are intersected in two OFDM symbols as shown in Fig. 3(c). For a block of $K$ OFDM symbols, the iterative algorithm proceeds as following:

$$
\begin{aligned}
& \text { 1: } \underline{Y}^{(k, 1)}={\underline{Y_{p}}}^{(k)} \\
& \text { 2: for } i=1: \mathrm{N}_{\text {iteration }} \text { do } \\
& \text { 3: } \quad \underline{\bar{\alpha}}_{L S}^{(k, i)}=\underline{\underline{M}} Y_{p}^{(k, i)} \\
& \text { 4: } \quad\left\{\hat{\alpha}_{l}^{(k, i)}\left[q T_{s}\right], \begin{array}{l}
k=2, \ldots, K-1 \\
q=-N_{g}, \ldots, N-1
\end{array}\right\}=\operatorname{interp}\left(\bar{\alpha}_{l_{L S}}^{(k, i)}, N+N_{g}\right) \\
& \text { 5: compute using (5) the channel matrix } \underline{\underline{\hat{H}}}^{(k, i)} \\
& \text { 6: remove the ICI of pilots from the received } \\
& \text { data subcarriers } \underline{Y}_{d}^{(k)} \text { in (31) } \\
& \text { 7: detection the data symbols }{\underline{\hat{X}_{d}}}^{(k, i)} \text { using SIS } \\
& \text { 8: } \quad \underline{Y}^{(k, i+1)}={\underline{Y_{p}}}^{(k)}-\underline{\hat{H}}_{p_{I}}^{(k, i)} \underline{\hat{X}}^{(k, i)} \\
& \text { 9: end for }
\end{aligned}
$$

where $\mathrm{N}_{\text {iteration }}$ is the number of iterations, interp denotes the interpolation Matlab function and, $i$ and $k$ represent the iteration number and the number of OFDM symbol in a block, respectively. Note that, the steps 3 to 6 are executed without considering the first and the last OFDM symbols (i.e., $k=2$ to $K-1$ ) in order to avoid limiting effects of interpolation.

\subsection{Mean Square Error (MSE) Analysis}

The MSE of the LS-estimator of $\underline{\bar{\alpha}}$ is defined by:

$$
m s e_{1}=E\left[\left(\underline{\bar{\alpha}}_{L S}-\underline{\bar{\alpha}}\right)^{H}\left(\underline{\bar{\alpha}}_{L S}-\underline{\bar{\alpha}}\right)\right]
$$

which gives (see Appendix D):

$$
m s e_{1}=\operatorname{Tr}\left(\underline{\underline{M}}\left(\underline{\underline{R_{1}}}+\sigma^{2} \underline{\underline{I}}_{N_{p}}\right) \underline{\underline{M}}^{H}\right)
$$

where the expression of the covariance matrix $R_{1}$ is detailed in Appendix D. Notice that if ICI are completely eliminated then, $R_{1}$ is matrix of zeros. Thus, (15) becomes:

$$
m s e_{1} \text { (without ICI) }=\sigma^{2} \operatorname{Tr}\left(\underline{\underline{M}} \underline{\underline{M}}^{H}\right)
$$

In general, mse $_{1}$ depends on the pilot positions and the multipath delays.
It is clear that our LS-estimator is unbiased. So, the CRAMER-RAO BOUND (CRB) [14] is an important criterion to evaluate how good the LS-estimator can be since it provides the MMSE bound among all unbiased estimators. We have shown in Appendix $\mathrm{C}$ that the Standard CRB (SCRB) for the estimator of $\underline{\bar{\alpha}}$ with ICI known is given by:

$$
\operatorname{SCRB}(\underline{\bar{\alpha}})=\frac{1}{S N R} \operatorname{Tr}\left(\left(\underline{\underline{F_{p}}}{ }^{H} \underline{\underline{X_{p}}}{ }^{H} \underline{\underline{X_{p}}} \underline{\underline{F_{p}}}\right)^{-1}\right)
$$

where $S N R=\frac{1}{\sigma^{2}}$ is the normalized signal to noise ratio. It is easy to show that

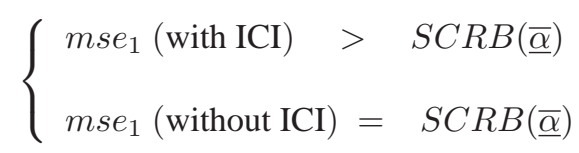

So, by iteratively estimating and removing the ICI $m s e_{1}$ will be closer to $S C R B(\underline{\bar{\alpha}})$.

The MSE of the assumption that $\underline{\underline{\alpha}}_{L S}$ is an estimation of $\underline{\alpha}_{c}$ is given by (see Appendix D):

$$
\begin{aligned}
m s e_{c} & =E\left[\left(\underline{\bar{\alpha}}_{L S}-\underline{\alpha}_{c}\right)^{H}\left(\underline{\bar{\alpha}}_{L S}-\underline{\alpha}_{c}\right)\right] \\
& =m s e_{1}+m s e_{2}+m s e_{12}+m s e_{21}
\end{aligned}
$$

where $m_{s e_{2}}$ is defined in (12) and, $m s e_{12}$ and $m s e_{21}$ are the cross-covariance terms, which are very negligible, given by (see Appendix D):

$$
\begin{aligned}
& m s e_{12}=E\left[\left(\underline{\bar{\alpha}}_{L S}-\underline{\bar{\alpha}}\right)^{H}\left(\underline{\bar{\alpha}}-\underline{\alpha}_{c}\right)\right]=\operatorname{Tr}\left(\underline{\underline{R}}^{\underline{M}^{H}}\right) \\
& m_{s e_{21}}=E\left[\left(\underline{\bar{\alpha}}-\underline{\alpha}_{c}\right)^{H}\left(\underline{\bar{\alpha}}_{L S}-\underline{\bar{\alpha}}\right)\right]=m s e_{12}^{*}
\end{aligned}
$$

where the matrix $R_{2}$ is computed in Appendix D.

The MSE of the multipath complex gain estimator at time $T_{s}$ is defined by:

$$
\begin{gathered}
\text { mse }_{T_{s}}=\sum_{k=2}^{K-1} \sum_{q=-N_{g}}^{N-1} E\left[\left(\underline{\hat{\alpha}}_{q}^{k}-\underline{\alpha}_{q}^{k}\right)^{H}\left(\underline{\hat{\alpha}}_{q}^{k}-\underline{\alpha}_{q}^{k}\right)\right] \\
\text { with } \underline{\alpha}_{q}^{k}=\left[\alpha_{1}^{k}\left(q T_{s}\right), \ldots, \alpha_{L}^{k}\left(q T_{s}\right)\right]^{T}
\end{gathered}
$$

For a large value $K$, assuming performant interpolator and respecting sampling theorem in time domain $\left(f_{d} T \leq\right.$ 0.5), we will have (see Appendix D):

$$
m s e_{T_{s}} \approx m s e_{c}
$$

We now study the MSE of the multipath complex gain estimator and the interpolation method versus the 


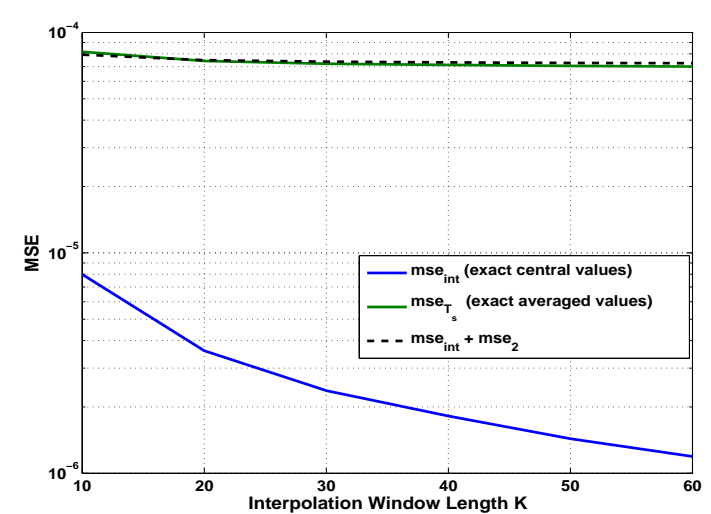

Figure 4. MSE of the complex gain estimator in terms of $K$ for $f_{d} T=0.1$

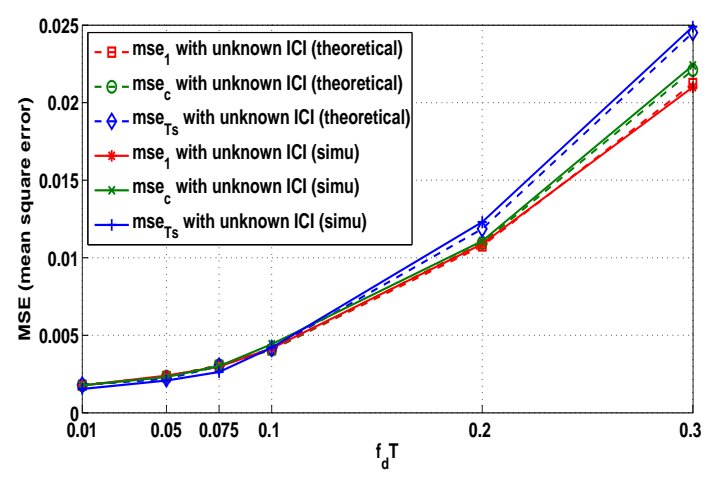

Figure 5. Comparison between MSE for $S N R=20 d B$

OFDM block length $K$. Fig. 4 gives the $m s e_{T_{s}}$ (with exact averaged values) and the mse $_{\text {int }}$ (with exact central values) for $f_{d} T=0.1$. We notice that the interpolation error $m s e_{i n t}$ decreases with $K$, while the error of the estimator $m s e_{T_{s}}$ is constant whatever the interpolation window length $K$. This is due to $\mathrm{mse}_{2}$ (MSE between the central value $\underline{\alpha}_{c}$ and the averaged value $\underline{\underline{\alpha}}$ ) which is dominant with respect to the interpolation error $m s e_{i n t}$. Moreover, we verify that $m s e_{T_{s}} \approx m s e_{i n t}+m s e_{2}$. This means that the cross-covariance terms are very negligible. So, in general, we may say that the interpolation window length $K$ is not necessary to be large and it suffices to choose $K$ such that $K . T=T_{\text {coh }}=\frac{1}{f_{d}}$ (i.e., $T_{\text {coh }}$ is the coherence time) in order to have strong correlation between samples $\alpha_{l}^{(n)}\left(q T_{s}\right)$. For example $f_{d} T=0.1, T_{\text {coh }}=10 T$, so we choose $K=10$.

\section{SIMULATION RESULTS}

In this section, we verify the theory by simulation and we test the performance of the iterative algorithm.
Table 1. Parameters of Channel

\begin{tabular}{|c|c|c|}
\hline \multicolumn{3}{|c|}{ Rayleigh Channel } \\
\hline Path Number & Average Power $(d B)$ & Normalized Delay \\
\hline 1 & -7.219 & 0 \\
\hline 2 & -4.219 & 0.4 \\
\hline 3 & -6.219 & 1 \\
\hline 4 & -10.219 & 3.2 \\
\hline 5 & -12.219 & 4.6 \\
\hline 6 & -14.219 & 10 \\
\hline
\end{tabular}

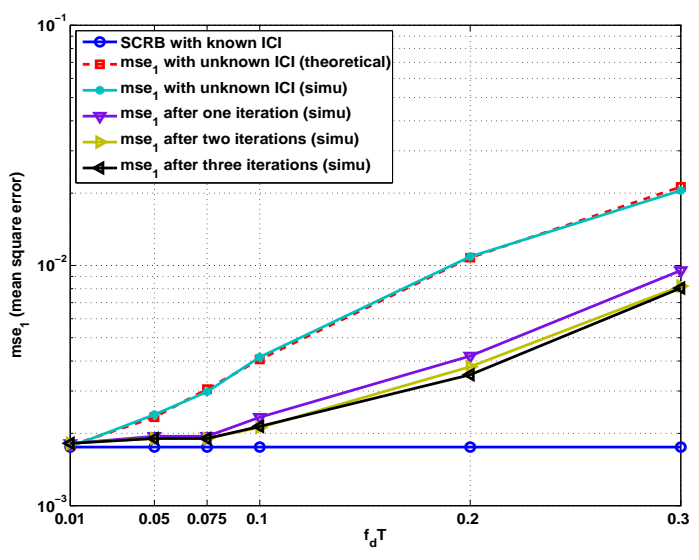

Figure 6. The MSE of the LS-estimator for $S N R=20 d B$

The mean square error (MSE) and the bit error rate (BER) performances in terms of the average signal-tonoise ratio (SNR) [9] [8] and maximum Doppler spread $f_{d} T$ (normalized by $1 / T$ ) for Rayleigh channel are examined. The normalized channel model is Rayleigh as recommended by GSM Recommendations 05.05 [12] [13], with parameters shown in the table below $\left(\frac{1}{T_{s}}=2 \mathrm{MHz}\right)$. A 4QAM-OFDM system with normalized symbols, $N=$ 128 subcarriers, $N_{g}=\frac{N}{8}$ subcarriers, $N_{p}=16$ pilots (i.e., $L_{f}=8$ ) and $K=10$ OFDM symbols in each block is used. (note that $(S N R) d B=\left(\frac{E_{b}}{N_{0}}\right) d B+3 d B$ ). These parameters are selected in order to have some concordance with the standard WiMax IEEE802.16e (same spacing between subcarriers about $10 \mathrm{KHz}$ for a carrier frequency $f_{c}=2.5 G H z$ and same rate between the symbol duration and the guard time). The BER performance of our iterative algorithm is evaluated under a relatively rapid time-varying channel such as $f_{d} T=0.05$ and $f_{d} T=0.1$ corresponding to a vehicle speed $V_{m}=140 \mathrm{~km} / \mathrm{h}$ and $V_{m}=280 \mathrm{~km} / \mathrm{h}$, respectively, for $f_{c}=5 \mathrm{GHz}$.

Fig. 5 shows the MSE in terms of $f_{d} T$ for $S N R=$ $20 d B$. It is observed that, with all ICI, the MSE obtained by simulation agrees with the theoretical value of MSE. We notice that the difference between $m s e_{T_{s}}$ and $m s e_{c}$ 


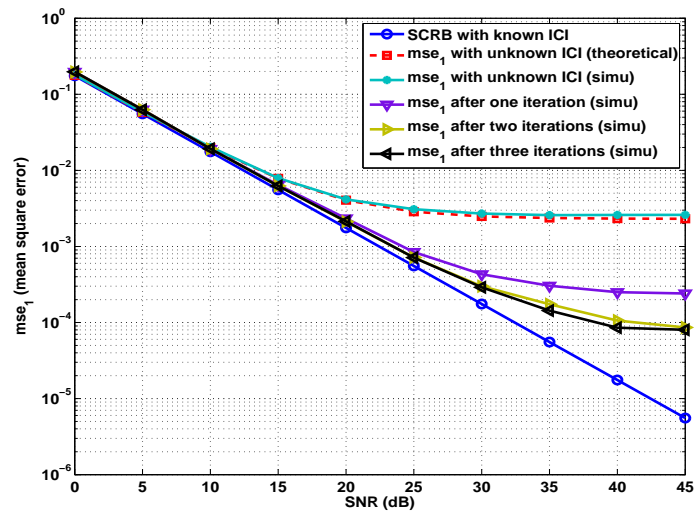

Figure 7. The MSE of the LS-estimator for $f_{d} T=0.1$

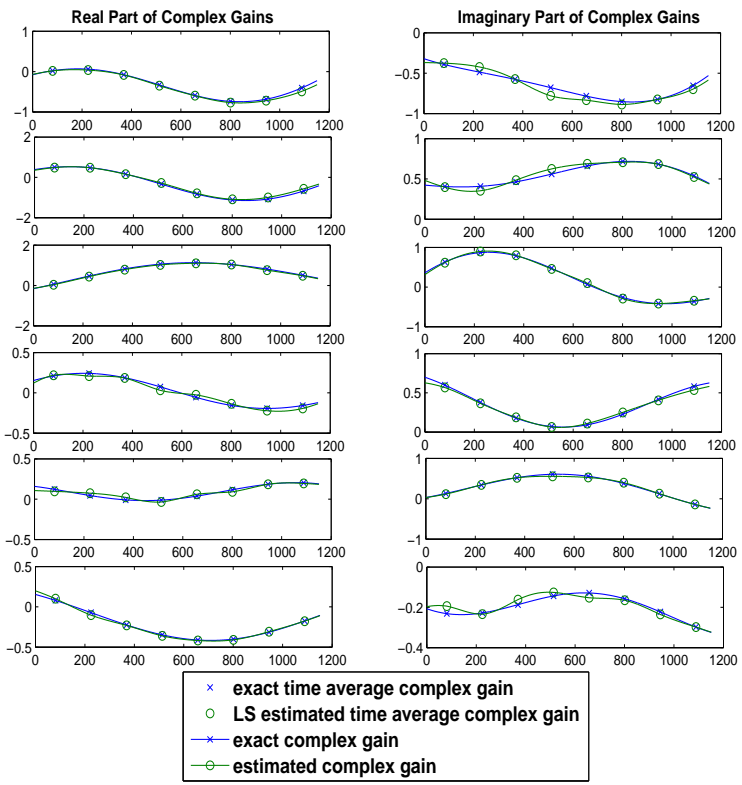

Figure 8. The LS estimated complex gain of six paths over 8 OFDM symbols after one iteration with $S N R=20 d B$ and $f_{d} T=0.1$

increases in terms of $f_{d} T$. This is due to the interpolation error which increases with $f_{d} T$. In short, we can say that $m s e_{T_{s}} \approx m s e_{c}$ and especially for $f_{d} T \leq 0.1$, which means our method is adequate over a block of $K$ OFDM symbols. We verify that $m s e_{2}$ is negligible with respect to $m s e_{1}$ (see Fig. 2 and 5) and especially for $f_{d} T \leq 0.2$, thus $m s e_{c} \approx m s e_{1}$

Fig. 6 gives the evolution of $m s e_{1}$ with the iterations in terms of $f_{d} T$ for $S N R=20 d B$. We notice that, with all ICI, $m s e_{1}$ is far from SCRB and when we commence to reduce the ICI, by improving the estimation of ICI at each iteration, $m_{s e}$ shows a significant improvement especially after the first iteration and approaches the SCRB for $f_{d} T \leq$

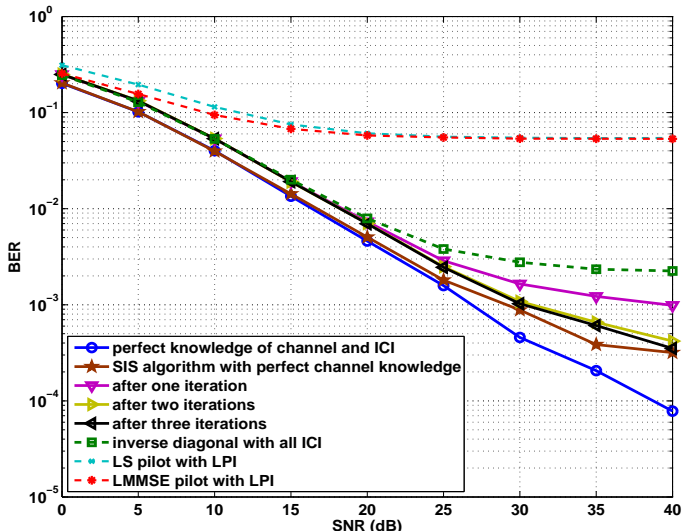

(a)

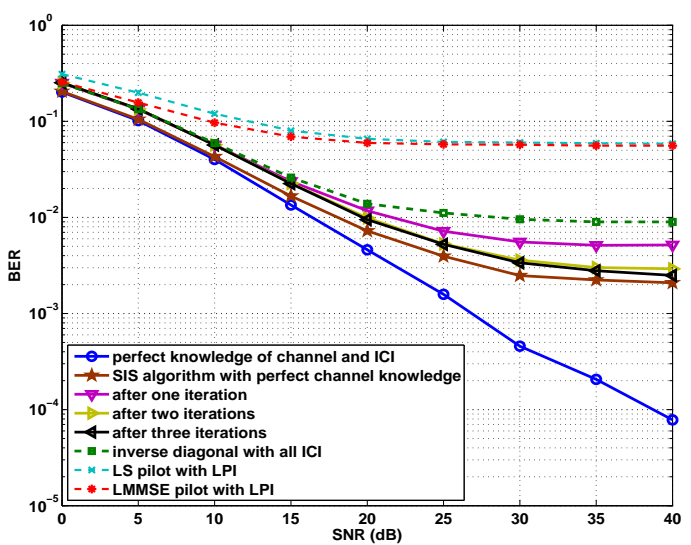

(b)

Figure 9. Comparison of BER: (a) $f_{d} T=0.05$; (b) $f_{d} T=0.1$

0.1 . However, by increasing $f_{d} T$, we show from $m s e_{2}$ given in Fig. 2 that $\underline{\bar{\alpha}}$ moves away from $\underline{\alpha}_{c}$. Hence, for $f_{d} T>0.1$, MSE of complex gains estimator is significant and the ICIs are not estimated and nor removed perfectly.

Fig. 7 shows the evolution of $m s e_{T_{s}} \approx m s e_{1}$ with the iterations in terms of $S N R$ for $f_{d} T=0.1$. Note that the xaxis represents the time axis normalized with respect to the sample time $T_{s}$. After one iteration, a great improvement is realized and $m s e_{1}$ is very close to the SCRB especially in low and moderate SNR regions. This is because at low SNR, the noise is dominant with respect to the ICI level, and at high SNR ICI is not completely removed due to the data symbol detection error.

For illustration, Fig. 8 gives the real and the imaginary parts of the exact and estimated (after one iteration) multipath complex gain. This is done for one channel realization over 8 OFDM symbols with $S N R=20 d B$ and $f_{d} T=0.1$. Notice how good is the estimation of multipath complex gains for rapidly changing channels. 


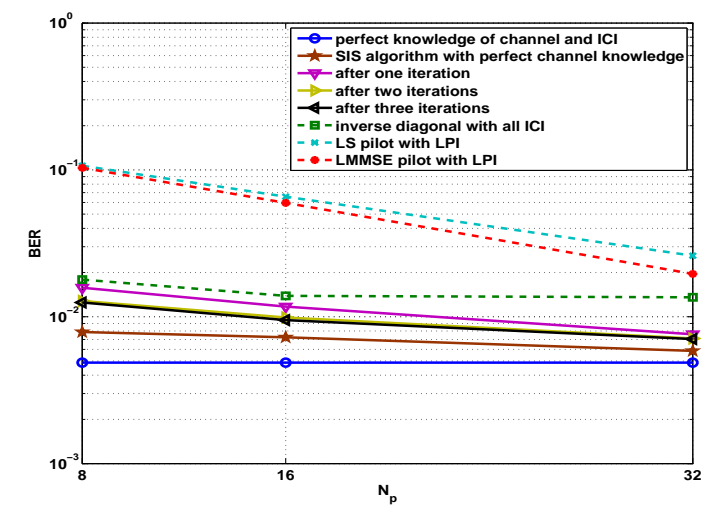

Figure 10. Comparison of BER for $f_{d} T=0.1$ and $S N R=$ $20 d B$

Fig. 9 gives the BER performance of our proposed iterative algorithm, compared to conventional methods (LS and LMMSE criteria with LPI in frequency domain) [6] [8] and SIS algorithm with perfect channel knowledge for $f_{d} T=0.05$ and $f_{d} T=0.1$. As reference, we also plotted the performance obtained with perfect knowledge of channel and ICI. This result shows that, with all ICI, our algorithm performs better than the conventional methods. Moreover, when we start removing ICI our iterative algorithm offers an improvement in BER after each iteration because the estimation of ICI is improved during each iteration. After two iterations, a significant improvement occurs; the performance of our algorithm and the SIS algorithm with perfect channel knowledge are very close. At a high SNR, it is normal to not reach the performance obtained with perfect knowledge of channel and ICI because we have an error floor due to the data symbol detection error.

Fig. 10 gives the BER in terms of $N_{p}$ for $f_{d} T=0.1$ and $S N R=20 d B$. It is obvious that when using more pilots, performance will be better. Moreover, the results show that, with less pilots and without interference suppression, our algorithm performs better than the conventional methods and becomes better with starting interference suppression.

Fig. 11 shows the BER performance of our proposed iterative algorithm, for $N_{c}=2$ and $f_{d} T=0.1$ with IEEE802.11a standard channel coding [18]. The convolutional encoder has a rate of $1 / 2$, and its polynomials are $P_{0}=133_{8}$ and $P_{1}=171_{8}$ and the interleaver is a bitwise block interleaver with 16 rows and 14 columns. It can clearly be seen that a significant improvement in BER occurs with channel coding, and that for high SNR there is always an error floor due to data symbol detection errors.

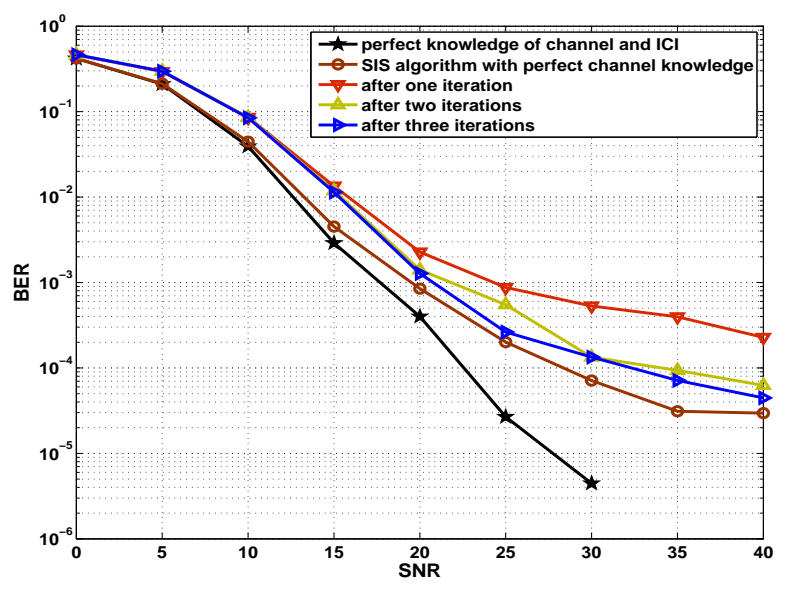

Figure 11. Comparison of BER, in the case of the IEEE802.11a convolutional code, for $f_{d} T=0.1$

\section{CONCLUSION}

In this paper, we have analyzed an iterative algorithm to estimate multipath complex gains and mitigate the intersub-carrier-interference (ICI) for OFDM systems. The rapid time-variation complex gains are tracked by exploiting that the delays are assumed invariant (over several symbols) and perfectly estimated. Theoretical analysis and simulation results of our iterative algorithm show that by estimating and removing the ICI at each iteration, multipath complex gains estimation and coherent demodulation can have a great improvement especially after the first iteration for high realistic Doppler spread. Moreover, our algorithm performs better than the conventional methods and its BER performance is very close to the performance of SIS algorithm with perfect channel knowledge.

\section{A. RECEIVED OFDM SYMBOL}

From equations (1) and (3) the received signal of the $n$th OFDM symbol at the output of the low pass receiver filter $g_{r}(t)$ is given by:

$y_{(n)}(t)=\sum_{d=-N_{g}}^{N-1} \sum_{l=1}^{L} x_{(n)}[d] \alpha_{l}(t) \beta\left(t-d T_{s}-n T-\tau_{l} T_{s}\right)+w(t)$

where $\beta(t)=\left(g_{t} \otimes g_{r}\right)(t)$. After A/D conversion and removing the cyclic prefix, the $\mathrm{N}$ received samples are given by: 


$$
\begin{gathered}
y_{(n)}[q]=\left.y_{(n)}(t)\right|_{t=q T_{s}+n T}= \\
\sum_{d=-N_{g}}^{N-1} \sum_{l=1}^{L} x_{(n)}[d] \alpha_{l}^{(n)}(q T s) \beta\left(\left(q-d-\tau_{l}\right) T_{s}\right)+w_{(n)}(q T s)
\end{gathered}
$$

where $q \in[0, N-1]$. Using (2), the $\mathrm{N}$ samples of the FFT output are given by:

$$
\begin{gathered}
Y_{(n)}[k]=\sum_{q=0}^{N-1} y_{(n)}[q] e^{-j 2 \pi \frac{k q}{N}}= \\
\frac{1}{N} \sum_{m=-\frac{N}{2}}^{\frac{N}{2}-1} X_{(n)}[m]\left(\sum_{q=0}^{N-1} e^{-j 2 \pi \frac{k q}{N}} \sum_{d=-N_{g}}^{N-1} \sum_{l=1}^{L} \alpha_{l}^{(n)}(q T s)\right. \\
\left.\beta\left(\left(q-d-\tau_{l}\right) T_{s}\right) e^{j 2 \pi \frac{m d}{N}}\right)+W_{(n)}[k]
\end{gathered}
$$

where $\quad k \in\left[-\frac{N}{2}, \frac{N}{2}-1\right] \quad$ and $\quad W_{(n)}[k]=$ $\sum_{q=0}^{N-1} w_{(n)}\left(q T_{s}\right) e^{-j 2 \pi \frac{k q}{N}}$.

As a result, for $\tau_{l}<N_{g}$, we have:

$$
\begin{gathered}
\sum_{u=0}^{N_{g}} \sum_{l=1}^{L} \alpha_{l}^{(n)}[q T s] \beta\left(\left(u-\tau_{l}\right) T_{s}\right) e^{-j 2 \pi \frac{m u}{N}} \approx \\
\sum_{l=1}^{L} \alpha_{l}^{(n)}(q T s) G_{t}[m] G_{r}[m] e^{-j 2 \pi \frac{m}{N} \tau_{l}}
\end{gathered}
$$

Notice that strict equality would hold if $u$ varies from $-\infty$ to $+\infty$. Inserting (23) into (22) yields the results given in equations (4) and (5).

\section{B. CLOSEST SAMPLE TO TIME AVERAGED COMPLEX GAIN}

Let $\underline{\alpha}_{d}=\left[\alpha_{1}\left(d T_{s}\right), \ldots, \alpha_{L}\left(d T_{s}\right)\right]^{T}$ be a vector of samples of the complex gains taken at the time position $d \in$ $[0, N-1]$ during the effective duration of the OFDM symbol. The MSE between $\underline{\bar{\alpha}}$ and $\underline{\alpha}_{d}$ is defined as:

$$
\begin{gathered}
m s e[d]=E\left[\left(\underline{\bar{\alpha}}-\underline{\alpha}_{d}\right)^{H}\left(\underline{\bar{\alpha}}-\underline{\alpha}_{d}\right)\right]= \\
\sum_{l=1}^{L} E\left[{\overline{\alpha_{l}}}_{\alpha_{l}}^{*}-\overline{\alpha_{l}} \alpha_{l}^{*}\left(d T_{s}\right)-\alpha_{l}\left(d T_{s}\right){\overline{\alpha_{l}}}^{*}+\alpha_{l}\left(d T_{s}\right) \alpha_{l}^{*}\left(d T_{s}\right)\right]
\end{gathered}
$$

Since $\alpha_{l}(t)$ is wide-sense stationary (WSS) narrow-band complex Gaussian processes with the so-called Jakes'

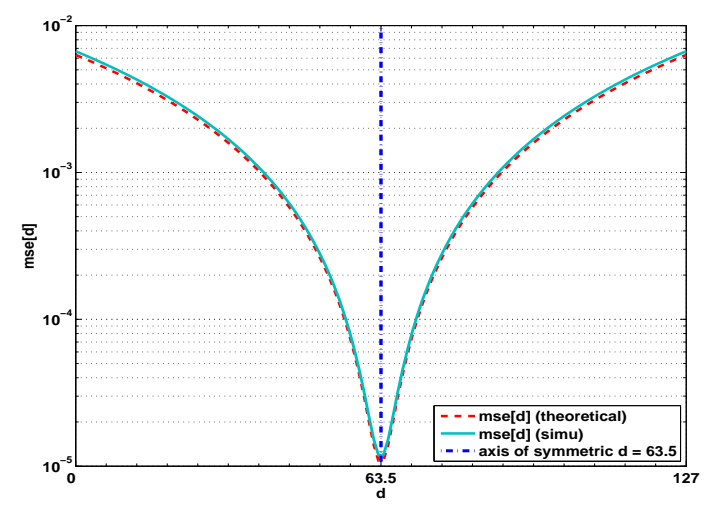

Figure 12. $m s e[d]$ with $N=128$ and $f_{d} T=0.1$

power spectrum [10] then:

$E\left[\alpha_{l}\left(q_{1} T_{s}\right) \alpha_{l}^{*}\left(q_{2} T_{s}\right)\right]=\sigma_{\alpha_{l}}^{2} J_{0}\left(2 \pi f_{d} T_{s}\left(q_{1}-q_{2}\right)\right)$

Using (24), we can calculate $m s e[d]$ as:

$$
\begin{aligned}
m s e[d]= & \sum_{l=1}^{L} \sigma_{\alpha_{l}}^{2}\left(\frac{1}{N^{2}} \sum_{q_{1}=0}^{N-1} \sum_{q_{2}=0}^{N-1} J_{0}\left(2 \pi f_{d} T_{s}\left(q_{1}-q_{2}\right)\right)\right. \\
& \left.-\frac{2}{N} \sum_{q=0}^{N-1} J_{0}\left(2 \pi f_{d} T_{s}(q-d)\right)+1\right)
\end{aligned}
$$

To find the closest $\underline{\alpha}_{d}$ to $\underline{\bar{\alpha}}$, we need to find $d_{\min }$ that minimizes $m s e[d]$. By using the derivative formula of the Bessel function defined as: $J_{0}^{\prime}(t)=-J_{1}(t)$, we can calculate the derivative of $m s e[d]$ as:

$$
\begin{aligned}
m s e^{\prime}[d] & =-\frac{4 \pi f_{d} T_{s}}{N} \sum_{l=1}^{L} \sigma_{\alpha_{l}}^{2} \sum_{q=0}^{N-1} J_{1}\left(2 \pi f_{d} T_{s}(q-d)\right) \\
& =-\frac{4 \pi f_{d} T_{s}}{N} \sum_{l=1}^{L} \sigma_{\alpha_{l}}^{2} \sum_{u=-d}^{N-1-d} J_{1}\left(2 \pi f_{d} T_{s} u\right)
\end{aligned}
$$

Since $J_{1}(t)$ is an odd function then, the solution of the equation $m s e^{\prime}[d]=0$ is obtained when the interval of the index $u$ in (25) is centered at zero, thus $d=$ $\frac{N}{2}-\frac{1}{2}$ (not integer). It is easy to show that $m s e[d]$ is symmetric with respect to $d=\frac{N}{2}-\frac{1}{2}$ axis then, $d_{\min }=$ $\frac{N}{2}-1$ or $\frac{N}{2}$. We denote the minimum of $m s e[d]$ by $m s e_{2}=m s e\left[d_{\min }\right]$. For illustration, Fig. 12 gives the curve of $m s e[d]$ (theoretical and monte-carlo simulation) for $N=128$ and $f_{d} T=0.1$. It is well observed that $d=$ 63.5 is an axis of symmetric and $d_{\min }=63$ or 64 . 


\section{CRB FOR THE ESTIMATOR OF $\underline{\bar{\alpha}}$}

Assuming $\underline{I C I_{p}}=\underline{H_{p_{I}}} \underline{X}$ in (8) are known then, the vector $Y_{p}$ for a given $\underline{\bar{\alpha}}$ is complex Gaussian with mean vector $\underline{\underline{m}}=\underline{\underline{X_{p}}} \underline{\underline{F_{p}}} \underline{\underline{\alpha}}+\underline{I C I_{p}}$ and covariance matrix $\underline{\underline{S_{1}}}=$ $\sigma^{2} \underline{\underline{I}}_{N_{p}}$. Thus, the probability density function $p\left(\underline{Y_{p}} \underline{\overline{\bar{\alpha}}}\right)$ is defined as:

$$
p\left(\underline{Y_{p}} \mid \underline{\bar{\alpha}}\right)=\frac{1}{\left|2 \pi \underline{\underline{S_{1}}}\right|} e^{\left.-\frac{1}{2}\left(\underline{Y_{p}}-\underline{m}\right)^{H} \underline{\underline{S_{1}}}-1 \underline{Y_{p}}-\underline{m}\right)}
$$

Since $\underline{\bar{\alpha}}$ is a complex Gaussian vector with zero mean and covariance matrix $\underline{\underline{S_{2}}}$ then, the probability density function of $\underline{\bar{\alpha}}$ is defined as:

$$
p(\underline{\bar{\alpha}})=\frac{1}{\left|2 \pi \underline{\underline{S_{2}}}\right|} e^{-\frac{1}{2} \underline{\bar{\alpha}}^{H}{\underline{\underline{S_{2}}}}^{-1} \underline{\bar{\alpha}}}
$$

where $\underline{S_{2}}$ is a diagonal matrix of elements $S_{2}[l, l]$, with $l \in[1, \overline{\bar{L}]}$, given by:

$S_{2}[l, l]=E\left[\overline{\alpha_{l}}{\overline{\alpha_{l}}}^{*}\right]=\frac{\sigma_{\alpha_{l}}^{2}}{N^{2}} \sum_{q_{1}=0}^{N-1} \sum_{q_{2}=0}^{N-1} J_{0}\left(2 \pi f_{d} T_{s}\left(q_{1}-q_{2}\right)\right)$

The Standard CRB (SCRB) and the Bayesian CRB (BCRB) for the estimator of $\underline{\bar{\alpha}}$ are defined as [14]:

$$
\begin{aligned}
& \operatorname{SCRB}(\underline{\bar{\alpha}})=\operatorname{Tr}\left(\left(-E\left[\frac{\partial^{2}}{\partial \underline{\bar{\alpha}}^{2}} \ln \left(p\left(\underline{Y_{p}} \mid \underline{\bar{\alpha}}\right)\right)\right]\right)^{-1}\right) \\
& B C R B(\underline{\bar{\alpha}})=\operatorname{Tr}\left(\left(-E\left[\frac{\partial^{2}}{\partial \overline{\underline{\alpha}}^{2}} \ln \left(p\left(\underline{Y_{p}}, \underline{\bar{\alpha}}\right)\right)\right]\right)^{-1}\right)
\end{aligned}
$$

where $p\left(Y_{p}, \underline{\bar{\alpha}}\right)=p\left(Y_{p} \mid \underline{\bar{\alpha}}\right) p(\underline{\bar{\alpha}})$ is the joint probability density function of $\overline{Y_{p}}$ and $\underline{\bar{\alpha}}$ and, the expectation is taken over $Y_{p}$ and $\bar{\alpha}$. Notice that SCRB and BCRB are for the estimation of deterministic and random variables, respectively.

The results of the second derivatives of $\ln \left(p\left(\underline{Y_{p}} \mid \underline{\bar{\alpha}}\right)\right)$ and $\ln \left(p\left(\underline{Y_{p}}, \underline{\bar{\alpha}}\right)\right)$ with respect to $\underline{\bar{\alpha}}$ are given by:

$$
\begin{aligned}
& \frac{\partial^{2}}{\partial \underline{\bar{\alpha}}^{2}} \ln \left(p\left(\underline{Y_{p}} \mid \underline{\bar{\alpha}}\right)\right)=-\underline{\underline{F_{p}}}{ }^{H} \underline{\underline{X_{p}}}{ }^{H} \underline{\underline{S_{1}}}{ }^{-1} \underline{\underline{X_{p}}} \underline{\underline{F_{p}}} \\
& \frac{\partial^{2}}{\partial \underline{\bar{\alpha}}^{2}} \ln \left(p\left(\underline{Y_{p}}, \underline{\bar{\alpha}}\right)\right)=-\underline{\underline{F_{p}}}{ }^{H} \underline{\underline{X_{p}}} \underline{\underline{S_{1}}}{ }^{-1} \underline{\underline{X_{p}}} \underline{\underline{F_{p}}}-{\underline{\underline{S_{2}}}}^{-1}
\end{aligned}
$$

Hence, substituting (27) in (26) yields:

$$
\begin{aligned}
& \operatorname{SCRB}(\underline{\underline{\alpha}})=\sigma^{2} \operatorname{Tr}\left(\left(\underline{\underline{F_{p}}} \underline{\underline{X_{p}}}{ }^{H} \underline{\underline{X_{p}}} \underline{\underline{F_{p}}}\right)^{-1}\right) \\
& \operatorname{BCRB}(\underline{\bar{\alpha}})=\operatorname{Tr}\left(\left(\frac{1}{\sigma^{2}} \underline{\underline{F_{p}}} \underline{\underline{X_{p}}} \underline{\underline{X_{p}}} \underline{\underline{F_{p}}}+{\underline{\underline{S_{2}}}}^{-1}\right)^{-1}\right)
\end{aligned}
$$

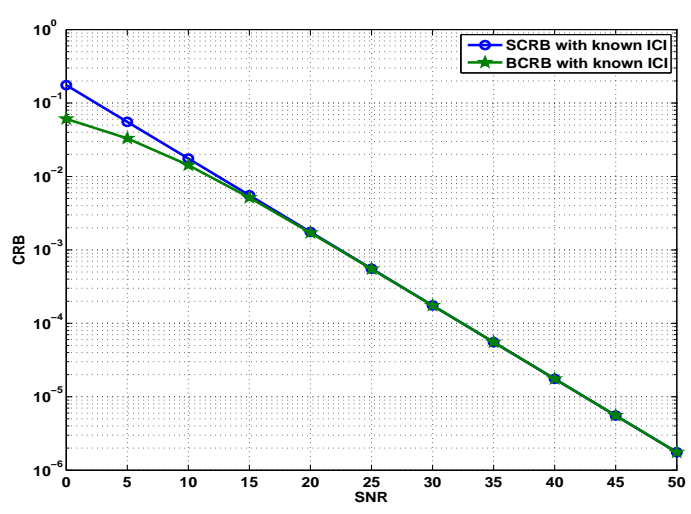

Figure 13. SCBR and BCRC with $N=128, N_{p}=16$ and $f_{d} T=0.1$

We notice that in our specific problem SCRB is independent of $\underline{\alpha}$. So, SCRB gives the lower bound if the priori distribution of $\underline{\bar{\alpha}}$ is not used in the estimation method, whereas BRCB takes this information into account. For illustration, Fig. 13 gives the SCRB and BCRB in terms of $S N R$ for the channel given in Table 1, $N=128, N_{p}=$ 16 and $f_{d} T=0.1$. It is observed that there is a small difference between SCRB and BCRB at low $S N R$. So, we can compare the MSE of our LS-estimator of $\underline{\alpha}$ to SCRB instead of BCRB.

Moreover, with known ICI, the optimal estimators of deterministic $\underline{\bar{\alpha}}$ and random (Gaussian) $\underline{\bar{\alpha}}$ are LS-estimator and maximum likelihood (ML) estimator, respectively. In our algorithm, the LS-estimator was used (considering $\underline{\bar{\alpha}}$ deterministic) because it requires less information compared to ML-estimator.

\section{MEAN SQUARE ERROR OF COMPLEX GAINS ESTIMATOR}

The MSE of the LS-estimator of $\underline{\bar{\alpha}}$ is given by:

$$
\begin{aligned}
m s e_{1} & =E\left[\left(\underline{\underline{\alpha}}_{L S}-\underline{\bar{\alpha}}\right)^{H}\left(\underline{\bar{\alpha}}_{L S}-\underline{\bar{\alpha}}\right)\right] \\
& =\operatorname{Tr}\left(\underline{\underline{M}}\left(\underline{\underline{R_{1}}}+\sigma^{2} \underline{\underline{I}}_{N_{p}}\right) \underline{\underline{M}}^{H}\right)
\end{aligned}
$$

where $\underline{\underline{R_{1}}}=E\left[\underline{\underline{H_{p_{I}}}} \underline{X} \underline{X}^{H} \underline{\underline{H_{p_{I}}{ }^{H}}}\right]$ and the expectation is taken over the data symbols, the noise and the complex gains, since the noise and the ICIs are uncorrelated. The term $\underline{I C I_{p}}=\underline{H_{p_{I}}} \underline{X}$ can be written as the sum of two components:

$$
\underline{I C I_{p}}=\underline{\underline{H_{p p}}} \underline{\underline{X_{p}}}+\underline{\underline{H_{d d}}} \underline{\underline{X_{d}}}
$$

where $X_{d}$ is the data symbols and, $H_{p p}$ and $\underline{\underline{H_{d d}}}$ are a 


$$
\begin{aligned}
& R_{p p}[k, m]=E\left[\sum_{\substack{u_{1}=p_{0} \\
u_{1} \neq k}}^{p_{N_{p}-1}} \sum_{u_{2} \neq p_{0}}^{p_{N_{p}-1}} X\left[u_{1}\right] X^{*}\left[u_{2}\right] H\left[k, u_{1}\right] H^{*}\left[m, u_{2}\right]\right] \\
& =\frac{1}{N^{2}} \sum_{u_{1}=p_{0}}^{p_{N_{p}-1}} \sum_{u_{2}=p_{0}}^{p_{N_{p}-1}} \sum_{l=1}^{L} \sigma_{\alpha_{l}}^{2} X\left[u_{1}\right] X^{*}\left[u_{2}\right] e^{-j 2 \pi \frac{u_{1}-u_{2}}{N}} \tau_{l} \sum_{q_{1}=0}^{N-1} \sum_{q_{2}=0}^{N-1} e^{j 2 \pi \frac{\left(u_{1}-k\right) q_{1}-\left(u_{2}-m\right) q_{2}}{N}} J_{0}\left(2 \pi f_{d} T_{s}\left(q_{1}-q_{2}\right)\right) \\
& R_{d d}[k, m]=E\left[\sum_{\substack{u=-\frac{N}{2} \\
u \neq p_{s}}}^{\frac{N}{2}-1} H[k, u] H^{*}[m, u]\right] \\
& =\sum_{l=1}^{L} \sigma_{\alpha_{l}}^{2}\left(\delta_{k, m}-\frac{N_{p}}{N^{2}} \sum_{q_{1}=0}^{N-1} \sum_{q_{2}=0}^{N-1}(-1)^{q_{1}-q_{2}} e^{-j 2 \pi \frac{k q_{1}-m q_{2}}{N}} J_{0}\left(2 \pi f_{d} T_{s}\left(q_{1}-q_{2}\right)\right) \delta_{0,\left(q_{1}-q_{2}\right)_{N_{p}}}\right)
\end{aligned}
$$

$$
\begin{aligned}
R_{2}[l, k] & =E\left[\sum_{\substack{u=p_{0} \\
u \neq k}}^{p_{N_{p}-1}} X^{*}[u] H^{*}[k, u]\left(\bar{\alpha}_{l}-\alpha_{l}\left[\frac{N}{2} T_{s}\right]\right)\right] \\
& =\frac{\sigma_{\alpha_{l}}^{2} \sum_{N^{2}-1}^{p^{2}}}{N_{u=p_{0}}} X^{*}[u] e^{j 2 \pi \frac{u}{N} \tau_{l}} \sum_{q_{1}=0}^{N-1} \sum_{q_{2}=0}^{N-1} e^{-j 2 \pi \frac{(u-k) q_{1}}{N}}\left(J_{0}\left(2 \pi f_{d} T_{s}\left(q_{1}-q_{2}\right)\right)-J_{0}\left(2 \pi f_{d} T_{s}\left(q_{1}-\frac{N}{2}\right)\right)\right)
\end{aligned}
$$

elements given by:

$$
\begin{aligned}
& H_{p p}[k, m]= \begin{cases}H[k, m] & \text { if } k, m \in \mathcal{P} \\
0 & \text { if } k=m\end{cases} \\
& H_{d d}[k, m]=H[k, m] \text { if } k \in \mathcal{P}, m \in\left[-\frac{N}{2}, \frac{N}{2}-1\right]-\mathcal{P}
\end{aligned}
$$

Hence, the matrix $\underline{\underline{R_{1}}}$ becomes: $\underline{R_{1}}=\underline{R_{p p}}+\underline{\underline{R_{d d}}}$ where $\quad \underline{\underline{R_{p p}}}=E\left[\underline{\underline{H_{p p}}} \underline{\underline{X_{p}}} \underline{X_{p}{ }^{H}} \underline{\underline{H_{p p}}}{ }^{H}\right]$ and $\underline{\underline{\underline{R_{d d}}}}=$ $E\left[\underline{\underline{H_{d d}}} \underline{\overline{X_{d}}} \underline{X}_{d}{ }^{H} \underline{\underline{H_{d d}{ }^{H}}}\right]$, since the data symbols and the coefficients $H[k, m]$ are uncorrelated.

Since the data symbols are normalized (i.e. $E\left[\underline{X_{d}}{\underline{X_{d}}}^{H}\right]=\underline{\underline{I}}_{N-N_{p}}$ ) then, $\underline{\underline{R_{d d}}}=E\left[\underline{\underline{H_{d d}}}{\underline{\underline{H_{d d}}}}^{H}\right]$. Thus, the elements $R_{p p}[k, m]$ and $R_{d d}[k, m]$, with $k, m \in \mathcal{P}$, are given by (28), shown at the top of the present page.
The MSE of the assumption that $\bar{\alpha}_{L S}$ is an estimation of $\underline{\alpha}_{c}$ is given by:

$$
\begin{aligned}
m s e_{c} & =E\left[\left(\underline{\bar{\alpha}}_{L S}-\underline{\alpha}_{c}\right)^{H}\left(\underline{\bar{\alpha}}_{L S}-\underline{\alpha}_{c}\right)\right] \\
& =m s e_{1}+m s e_{2}+m s e_{12}+m s e_{21}
\end{aligned}
$$

where $m s e_{12}$ and $m s e_{21}$ are the cross-covariance terms given by:

$$
\begin{aligned}
m_{12} & =E\left[\left(\underline{\bar{\alpha}}_{L S}-\underline{\bar{\alpha}}\right)^{H}\left(\underline{\bar{\alpha}}-\underline{\alpha}_{c}\right)\right] \\
& =\operatorname{Tr}\left(\underline{\underline{R_{2}}} \underline{\underline{M}}^{H}\right) \\
m s e_{21} & =E\left[\left(\underline{\bar{\alpha}}-\underline{\alpha}_{c}\right)^{H}\left(\underline{\bar{\alpha}}_{L S}-\underline{\bar{\alpha}}\right)\right]=m s e_{12}^{*}
\end{aligned}
$$

where $\underline{\underline{R_{2}}}=E\left[\left(\underline{\bar{\alpha}}-\underline{\alpha}_{c}\right) \underline{X}^{H} \underline{H p p}^{H}\right]$ is a $L \times N_{p}$ matrix of elements $R_{2}[l, k]$, with $\left.l \in \overline{\overline{11, L}}\right]$ and $k \in \mathcal{P}$, given by (29), shown at the top of the present page. Notice that the elements of the matrix $R_{1}$ and $R_{2}$ depend on the known pilot symbols and the multipath $\overline{\overline{\text { delays. }}}$ 
The MSE of the multipath complex gain estimator at time $T_{s}$ is defined by:

$$
m \operatorname{se}_{T_{s}}=\sum_{k=2}^{K-1} \sum_{q=-N_{g}}^{N-1} E\left[\underline{e}_{q}^{k^{H}} \underline{e}_{q}^{k}\right]
$$

where $\quad \underline{e}_{q}^{k}=\underline{\hat{\alpha}}_{q}^{k}-\underline{\alpha}_{q}^{k}, \quad$ with $\quad \underline{\alpha}_{q}^{k}=$ $\left[\alpha_{1}^{k}\left(q T_{s}\right), \ldots, \alpha_{L}^{k}\left(q T_{s}\right)\right]^{T}$, is the error of the multipath complex gain estimator at time $T_{s}$. This error can be written as the sum of two errors:

$$
\underline{e}_{q}^{k}=\underline{e}_{c}^{k}+\underline{e}_{i n t}^{k}
$$

where $\underline{e}_{c}^{k}$ is the error due to the assumption that $\underline{\bar{\alpha}}_{L S}$ is an estimation of $\underline{\alpha}_{c}$ and $e_{i n t}{ }^{k}$ is the error due to the interpolation method which depends on the number $K$ of OFDM symbols in each bloc and the term $f_{d} T$.

For a large value $K$, assuming performant interpolator and respecting sampling theorem in time domain (i.e. $f_{d} T \leq 0.5$ ), we will have $\underline{e}_{i n t}{ }^{k} \approx[0, \ldots, 0]^{T}$, and then:

$$
m s e_{T_{s}} \approx m s e_{c}
$$

\section{E. SUCCESSIVE INTERFERENCE SUPPRESSION METHOD}

The received data subcarriers are given by:

$$
\underline{Y_{d}}=\underline{\underline{H_{d}}} \underline{X_{d}}+\underline{\underline{H_{d}^{\prime}}} \underline{X_{p}}+\underline{W_{d}}
$$

where $X_{d}$ the data transmitted, $Y_{d}$ the data received and $W_{d}$ the noise at data subcarrier positions are $(N-$ $\left.N_{p}\right) \overline{\times 1}$ vectors, $\underline{H_{d}}$ and $H_{d}^{\prime}$ are a $\left(N-N_{p}\right) \times\left(N-N_{p}\right)$ and $\left.\left(N-N_{p}\right) \times \overline{\overline{\left(N_{p}\right.}}\right)$ matrices, respectively, of elements given by:

$$
\begin{aligned}
& H_{d}[k, m]=H[k, m] \text { if } k, m \in\left[-\frac{N}{2}, \frac{N}{2}-1\right]-\mathcal{P} \\
& H_{d}^{\prime}[k, m]=H[k, m] \text { if } k \in\left[-\frac{N}{2}, \frac{N}{2}-1\right]-\mathcal{P}, m \in \mathcal{P}
\end{aligned}
$$

Note that in (30), the first component is the desired data term with ICI due to data symbols and the second component is the ICI term due to pilot symbols.

By successive interference suppression (SIS) scheme with optimal ordering and one tap frequency equalizer the data will be estimated. The optimal ordering is calculated from the large to the small magnitude of the diagonal elements of the data channel matrix $\underline{\underline{H_{d}}}$ and given by:

$$
\begin{aligned}
\mathcal{O}= & \left\{O_{1}, O_{2}, \ldots, O_{N-N_{p}} \mid\right. \\
& \left.i<j \text { if }\left\|H_{d}\left[O_{i}, O_{i}\right]\right\|>\left\|H_{d}\left[O_{j}, O_{j}\right]\right\|\right\}
\end{aligned}
$$

The detection algorithm can now be described as follows:

$$
\begin{aligned}
& \text { 1: } \mathcal{O}=\left\{O_{1}, O_{2}, \ldots, O_{N-N_{p}}\right\} \\
& \text { 2: } \underline{Y_{d[1]}^{\prime}}=\underline{Y_{d}^{\prime}}=\underline{Y_{d}}-\underline{\underline{H_{d}^{\prime}}} \underline{X_{p}} \\
& \text { 3: for } i=1: N-N_{p} \text { do } \\
& \text { 4: } \quad X e_{d}\left[O_{i}\right]=Y_{d[i]}^{\prime}\left[O_{i}\right] / H_{d}\left[O_{i}, O_{i}\right] \\
& \text { 5: } \quad \hat{X}_{d}\left[O_{i}\right]=Q\left(X e_{d}\left[O_{i}\right]\right) \\
& \text { 6: } \quad \underline{Y_{d[i+1]}^{\prime}}=\underline{Y_{d[i]}^{\prime}}-\hat{X}_{d}\left[O_{i}\right]\left(\underline{H_{d}}\right)_{O_{i}}
\end{aligned}
$$

7: end for

where $Y_{d}^{\prime}$ is the received data subcarriers without contribution from pilot subcarriers, $Q($.$) denotes the$ quantization operation appropriate to the constellation in use and $\left(H_{d}\right)_{O_{i}}$ denotes the $O_{i}$ th column of the data channel matrix $H_{d}$. Note that in our algorithm, we have used the minimum distance criterion as quantization method.

\section{REFERENCES}

1. H. Hijazi and L. Ros, "Time-varying Channel Complex Gains Estimation and ICI Suppression in OFDM Systems" in IEEE GLOBAL COMMUNICATIONS Conf., Washington, USA, 2007 to be appeared.

2. H. Hijazi, L. Ros and G. Jourdain, “ OFDM Channel Parameters Estimation used for ICI Reduction in time-varying Multipath channels" in EUROPEAN WIRELESS Conf., Paris, FRANCE, April 2007.

3. E. Simon, L. Ros and K. Raoof, "Synchronization over rapidly timevarying multipath channel for CDMA downlink RAKE receivers in Time-Division mode", in IEEE Trans. Vehicular Techno., vol. 56. no. 4, Jul. 2007

4. E. Simon and L. Ros," Adaptive multipath channel estimation in CDMA based on prefiltering and combination with a linear equalizer",14th IST Mobile and Wireless Communications Summit, Dresden, June 2005.

5. A. R. S. Bahai and B. R. Saltzberg, Multi-Carrier Dications: Theory and Applications of OFDM: Kluwer Academic/Plenum, 1999.

6. M. Hsieh and C. Wei, "Channel estimation for OFDM systems based on comb-type pilot arrangement in frequency selective fading channels" in IEEE Trans. Consumer Electron., vol.44, no. 1, Feb. 1998.

7. O. Edfors, M. Sandell, J. -J. van de Beek, S. K. Wilson, and P. o. Brejesson, "OFDM channel estimation by singular value decomposition" in IEEE Trans. Commun., vol. 46, no. 7, pp. 931-939, Jul. 1998. 
8. S. Coleri, M. Ergen, A. Puri and A. Bahai, "Channel estimation techniques based on pilot arrangement in OFDM systems" in IEEE Trans. Broad., vol. 48. no. 3, pp. 223-229 Sep. 2002.

9. B. Yang, K. B. Letaief, R. S. Cheng and Z. Cao, "Channel estimation for OFDM transmisson in mutipath fading channels based on parametric channel modeling" in IEEE Trans. Commun., vol. 49, no. 3, pp. 467-479, March 2001.

10. W. C. Jakes, Microwave Mobile Communications. Piscataway, NJ: IEEE Press, 1983.

11. R. Roy and T. Kailath, "ESPRIT-Estimation of signal parameters via rotational invariance techniques" in IEEE Trans. Acoust., Speech, Signal Processing, vol. 37, pp. 984-995, July 1989.

12. European Telecommunications Standards Institute, European Digital Cellular Telecommunication System (Phase 2); Radio Transmission and Reception, GSM 05.05, vers. 4.6.0, Sophia Antipolis, France, July 1993.

13. Y. Zahao and A. Huang, "A novel Channel estimation method for OFDM Mobile Communications Systems based on pilot signals and transform domain processing" in Proc. IEEE 47th Vehicular Techno. Conf., Phonix, USA, May 1997, pp. 2089-2093.
14. H. L. Van Trees, Detection, estimation, and modulation theory: Part I, Wiley, New York, 1968.

15. Z. Tang, R. C. Cannizzaro, G. Leus and P. Banelli, "Pilot-assisted time-varying channel estimation for OFDM systems" in IEEE Trans. Signal Process., vol. 55, pp. 2226-2238, May 2007.

16. S. Tomasin, A. Gorokhov, H. Yang and J.-P. Linnartz, "Iterative interference cancellation and channel estimation for mobile OFDM" in IEEE Trans. Wireless Commun., vol. 4, no. 1, pp. 238-245, Jan. 2005.

17. M. K. Ozdemir and H. Arslan, "Channel Estimation for Wireless OFDM Systems", IEEE Communications Surveys and Tutorials, vol. 9, pp. 18-48, Issue: 2, Second Quarter 2007.

18. Y. Tang, L. Qian, and Y. Wang, "Optimized software implementation of a full-fate IEEE 802.11a compliant digital baseband transmitter on a digital signal processor" in IEEE GLOBAL Telecommun. Conf., vol. 4, Nov. 2005

19. P. Hoher, S. Kaiser and P. Robertson, "Pilot-symbol-aided channel estimation in time and frequency" in Proceedings of IEEE Global Telecommunications Conference, Communication Theory Mini Conference, pp. 9096, Phoenix, USA, Nov. 1997. 


\section{AUTHORS' BIOGRAPHIES}

Hussein Hijazi received the Ph.D. degree in signal processing and communications from the Institut National Polytechnique de Grenoble (INPG), Grenoble, France, in 25 November 2008, where he is currently an Associate Professor. His dissertation focused on channel estimation in a high speed mobile receiver operating in an OFDM communication system. Prior to earning his MASTER ( Signal, Image, Speech, Telecom ) from INPG in 2005, he was awarded the Diploma in computer and communications engineering from the Lebanese University (Faculty of Engineering), Beyrouth, Lebanon, in 2004. His current research interests lie in the areas of signal processing and communications, including synchronisation, channel estimation and equalization algorithms for wireless digital communications.

Laurent Ros received the degree in electrical engineering from the "École Supérieure d'Électricité" (Supélec), Paris, France, in 1992 and the Ph.D. degree in signal processing and communications from the Institut National Polytechnique de Grenoble (INPG), Grenoble, France, in 2001. From 1993 to 1995, he was with France-Telecom R \& D center, Lannion, France, where he worked in the area of very low frequency transmissions for submarine applications, in collaboration with "Direction des Constructions Navales", Toulon, France. From 1995 to 1999, he was a Research and Development Team Manager at Sodielec, Millau, France, where he worked in the design of digital modems and audio codecs for telecommunication applications. Since 1999, he has joined the Gipsa-lab/DIS (ex "Laboratory of Image and Signal"), INPG, where he is currently an Associate Professor. His general research interests include synchronisation, time-varying channel estimation and equalization problems for wireless digital communications. 